\title{
Mapping of cis-acting expression quantitative trait loci in human scalp hair follicles
}

\author{
Marisol Herrera-Rivero ${ }^{1,2}$, Lara M. Hochfeld ${ }^{1}$, Sugirthan Sivalingam', Markus M. Nöthen ${ }^{1}$ and
}

Stefanie Heilmann-Heimbach ${ }^{1 *}$ (D)

\begin{abstract}
Background: The association of molecular phenotypes, such as gene transcript levels, with human common genetic variation can help to improve our understanding of interindividual variability of tissue-specific gene regulation and its implications for disease.
\end{abstract}

Methods: With the aim to capture the spectrum of biological processes affected by regulatory common genetic variants (minor allele frequency $\geq 1 \%$ ) in healthy hair follicles (HFs) from scalp tissue, we performed a genome-wide mapping of cis-acting expression quantitative trait loci (eQTLs) in plucked HFs, and applied these eQTLs to help further explain genomic findings for hair-related traits.

Results: We report 374 high-confidence eQTLs found in occipital scalp tissue, whose associated genes (eGenes) showed enrichments for metabolic, mitotic and immune processes, as well as responses to steroid hormones. We were able to replicate 68 of these associations in a smaller, independent dataset, in either frontal and/or occipital scalp tissue. Furthermore, we found three genomic regions overlapping reported genetic loci for hair shape and hair color. We found evidence to confirm the contributions of PADI3 to human variation in hair traits and suggest a novel potential candidate gene within known loci for androgenetic alopecia.

Conclusions: Our study shows that an array of basic cellular functions relevant for hair growth are genetically regulated within the HF, and can be applied to aid the interpretation of interindividual variability on hair traits, as well as genetic findings for common hair disorders.

Keywords: Hair follicle, Hair loss, Expression quantitative trait loci, Androgenetic alopecia, Single nucleotide polymorphisms

\footnotetext{
* Correspondence: sheilman@uni-bonn.de

${ }^{1}$ Institute of Human Genetics, University of Bonn, School of Medicine \&

University Hospital Bonn, 53127 Bonn, Germany

Full list of author information is available at the end of the article
}

(C) The Author(s). 2020 Open Access This article is licensed under a Creative Commons Attribution 4.0 International License, which permits use, sharing, adaptation, distribution and reproduction in any medium or format, as long as you give appropriate credit to the original author(s) and the source, provide a link to the Creative Commons licence, and indicate if changes were made. The images or other third party material in this article are included in the article's Creative Commons licence, unless indicated otherwise in a credit line to the material. If material is not included in the article's Creative Commons licence and your intended use is not permitted by statutory regulation or exceeds the permitted use, you will need to obtain permission directly from the copyright holder. To view a copy of this licence, visit http://creativecommons.org/licenses/by/4.0/ The Creative Commons Public Domain Dedication waiver (http://creativecommons.org/publicdomain/zero/1.0/) applies to the data made available in this article, unless otherwise stated in a credit line to the data. 


\section{Background}

Human hair traits show wide interindividual variability, which has been suggested to be largely determined by genetic factors $[1,2]$.

Systematic gene identification efforts for a growing number of quantitative and complex human traits have shown that the majority of associated genetic factors are located in non-coding genomic regions [3]. These variants most probably exert their functional effects through the tissuespecific modulation of the expression of trait-relevant genes. Expression quantitative trait loci (eQTL) analyses, that correlate sequence variation with gene expression data, have proven a valuable tool in terms of delineating the tissue-specific architecture(s) of gene regulation and predicting the impact that trait-associated variants exert on it [4]. This approach therefore bridges the gap between a genetic association finding and the underlying biological mechanism, and may provide crucial insights into disease development.

Increased knowledge of the genetic factors that contribute to variability of gene regulation in the human scalp hair follicle (HF) will aid the interpretation of genetic findings for hair-related traits and hair loss disorders, such as androgenetic alopecia (AGA). Moreover, the comparison of the regulatory architecture between HFs from different scalp areas may aid the understanding of the variable susceptibility of hair follicle subpopulations to hormonal hair loss. The aims of the present study were to: 1 ) perform a systematic mapping of eQTLs in the human hair follicle, and 2) evaluate the potential of these eQTLs in terms of the functional annotation of genetic loci that contribute to the development of hair-related traits and common diseases.

\section{Methods}

\section{Sample collection}

About 50 HFs were plucked from the occipital scalp of 100 (discovery sample), and the frontal and occipital scalp of 25 (replication sample, previously described in [5]) unrelated male volunteers. Peripheral venous blood samples were collected from all study participants. All volunteers were German residents of European descent, and showed a collective mean age of 27.9 years.

\section{Extraction of nucleic acids}

DNA was extracted from whole blood samples using the Chemagic Magnetic Separation Module I (Perkin Elmer Chemagen Technology Inc., Baesweiler, Germany). Total RNA was extracted from HFs using the RNeasy Micro Kit (Qiagen, Hilden, Germany), and the quality and quantity of the RNA were assessed using a BioAnalyzer 2100 (Agilent Technologies, Waldbronn, Germany), and a NanoDrop ND-1000 spectrophotometer (Peqlab Biotechnologie, Erlangen, Germany), respectively. Only total
RNA samples with an RNA integrity number (RIN) $\geq 8$ were further analyzed in the study.

\section{Array hybridization}

DNA extracts were hybridized onto the Human OmniExpress-12v1.0 bead array (Illumina, San Diego, CA, USA) $(N=100)$ or the Illumina PsychArray v1.0 $(N=25)$ for genome-wide genotyping, while RNA extracts from HFs were amplified and biotinylated using the TotalPrep ${ }^{\text {тм }}-96$ RNA Amplification Kit (Illumina, San Diego, CA, USA) prior to whole transcriptome profiling, performed on the Illumina HT-12v4 bead array.

\section{Preparation of genotype data}

SNP array raw data was initially analyzed using the Genotyping module within the GenomeStudio software (Illumina). Genotype calls were exported for basic quality control in PLINK v1.9 ([6]; www.cog-genomics.org/plink/1. 9/) to eliminate bad quality data (e.g. SNPs and individuals with high degree of missing data, very rare SNPs) prior to genotype imputation. Imputation was performed on the Michigan Imputation Server [7] using the 1000 Genomes Project Phase 3 v5 reference panel and Eagle v2.3 phasing [8]. Post-imputation data processing was performed using VCFtools [9] and quality control was carried out in PLINK 1.9. Briefly, only biallelic single nucleotide variants with high imputation quality score (Rsq) $>0.7$, minor allele frequency $(\mathrm{MAF}) \geq 1 \%$ and under Hardy-Weinberg equilibrium (HWE $p>1 \times 10^{-8}$ ) were further analyzed. A principal component analysis (PCA) was performed with PLINK to identify potential outlier samples and use the generated principal components (PCs) as covariates for eQTL analysis. Two final autosomal genotype datasets consisted of 5,887,234 SNPs and 98 individuals for the discovery sample, and 1,044,566 SNPs and 24 individuals for the replication sample.

\section{Preparation of gene expression data}

Raw data from the expression microarrays was initially analyzed using the Gene Expression module within the GenomeStudio software to generate calls and detection $p$-values. The probe-level gene expression data was exported for pre-processing by background correction, quantile normalization, log2 transformation, probe quality filtering and identification of potential outlier samples by PCA using R. Probes were considered expressed when showing a detection p-value $<0.01$ in at least $5 \%$ of the samples. Probe quality filtering included the retention of only "good" and "perfect" quality probes mapping to only one gene with a valid identifier, according to annotations retrieved from the illuminaHumanv4.db package [10]. Three final gene expression datasets consisted of 13,217 probes and 98 individuals for the discovery sample, and 13,091 probes from frontal scalp and 12, 
814 probes from occipital scalp and 24 individuals for the replication sample.

\section{eQTL mapping}

Genome-wide associations in cis (1 Mb window) between the expression levels in scalp HFs and SNP genotypes were tested using QTLtools [11]. Three covariate files prepared for the analyses included the first 10 (discovery sample) or 5 (replication sample) PCs for the genotype datasets and the first 10 or 5 PCs for the phenotype datasets. Initially, the full spectrum of eQTLs for each of all three gene expression datasets was identified through nominal pass analysis. After exploration of the nominal significant results $(p<0.05)$, only those eQTLs with false discovery rate $(\mathrm{FDR})<1 \times 10^{-4}$ in the discovery sample were considered true eQTLs, while all eQTLs with $p<0.01$ in the replication sample were retained for further analyses. To identify independent signals within our set of true eQTLs, a permutation pass analysis (1000 permutations), followed by a conditional pass analysis on grouped phenotypes (i.e. a gene-level output from the probe-level analysis) were applied.

\section{Variant annotation}

We explored reported eQTL effects for our independent HF eQTLs using the Variant Annotation tool from SNiPA (Single Nucleotide Polymorphisms Annotator) ([12]; http://snipa.org), noting whether or not the HF eQTL has been previously reported to have cis-eQTL effects in at least one tissue, and whether or not eQTL effects have been observed on the same gene as in our study. Additionally, we searched for trait associations of our true HF eQTLs that have been reported in the GWAS Catalog ([13]; https://www.ebi.ac.uk/gwas/).

\section{Replication of HF eQTLs}

The true eQTLs identified from the nominal pass analysis in the discovery sample were considered replicated when the same eQTL SNP (eSNP) was found associated to the same eGene, either through the same or a different probe, with $p<0.01$ in either frontal and/or occipital scalp areas from the replication sample.

\section{Differential eQTLs between frontal and occipital scalp}

To investigate differential regulatory effects between frontal and occipital scalp areas, non-overlapping eQTLs with $p$-value $<5 \times 10^{-5}$ were investigated in the replication sample. These included eSNPs that showed different effects between both datasets (i.e. association to a different eGene or opposing direction of the effect for the same SNP-gene pair; different-effect eQTLs), and those eQTLs that were unique to the frontal or occipital dataset (i.e. no overlaps in eSNPs or eGenes; region-specific eQTLs).

\section{Functional enrichment analysis}

The lists of eGenes obtained from the discovery sample and the differential eQTL analysis were submitted for enrichment analysis using the GENE2FUNC function of the Functional Mapping and Annotation of GenomeWide Association Studies (FUMA GWAS) platform ([14]; http://fuma.ctglab.nl/). Each analysis used the provided option to include all human genes as background, a significance cutoff of FDR $<0.05$ and a threshold of minimum overlapping genes with the gene-sets $=3$. In addition, a comparative analysis of biological pathways for the differential eQTLs was performed using the lists of the identified region-specific eGenes and the Gene Enrichment Compare function within the FunRich (Functional Enrichment analysis tool) software [15]. From the resulting pathway analysis using the default FunRich database, only frontal- and occipital-specific terms with at least 2 genes from the dataset present in the pathway term, and $p<0.01$ (from hypergeometric test) in one scalp area and $p>0.05$ in the other, were retained for the purposes of the present study.

\section{Overlaps with reported genetic findings for hair phenotypes}

To test the informativeness of our HF eQTLs for the interpretation of genetic findings for hair phenotypes, we used three sets of published genome-wide significant variants $\left(p<5 \times 10^{-8}\right)$ associated with (i) hair shape (discovery meta-analysis, supplementary table 2 from [1]), (ii) hair color (meta-analysis, supplementary table 2 from [2]), and (iii) AGA (reported lead SNPs from [16-26]). These three variant sets were subjected to analysis by the SNP2GENE function of FUMA GWAS to assign GWAS findings to genomic regions. The analysis for each phenotype was set to include SNPs from the 1000 Genomes Project (Phase 3, European population) [27] that are in linkage disequilibrium (LD) with the reported GWAS variants. LD blocks were set to include variants of minor allele frequency $\geq 0.01, r^{2} \geq 0.6$ and a distance $<500 \mathrm{~kb}$ for merging into a locus. Afterwards, we searched for overlaps between our true HF eQTLs and the resulting loci for hair shape, hair color and AGA.

\section{Results}

\section{Hair follicle eQTLs}

We identified almost 3 million nominally significant eQTL signals in HFs from the occipital scalp (Fig. 1a). However, when we plotted the $p$-values and distances to the transcription start site (TSS) of the probe for each top SNPprobe pair with FDR $<0.05$, we observed that a large number of SNPs were lying over $250 \mathrm{~kb}$ from the probe's TSS, suggesting many of these might actually be noise signals (false positives). For this reason, only eQTL-findings that showed an FDR $<1 \times 10^{-4}$ were considered the likely true 

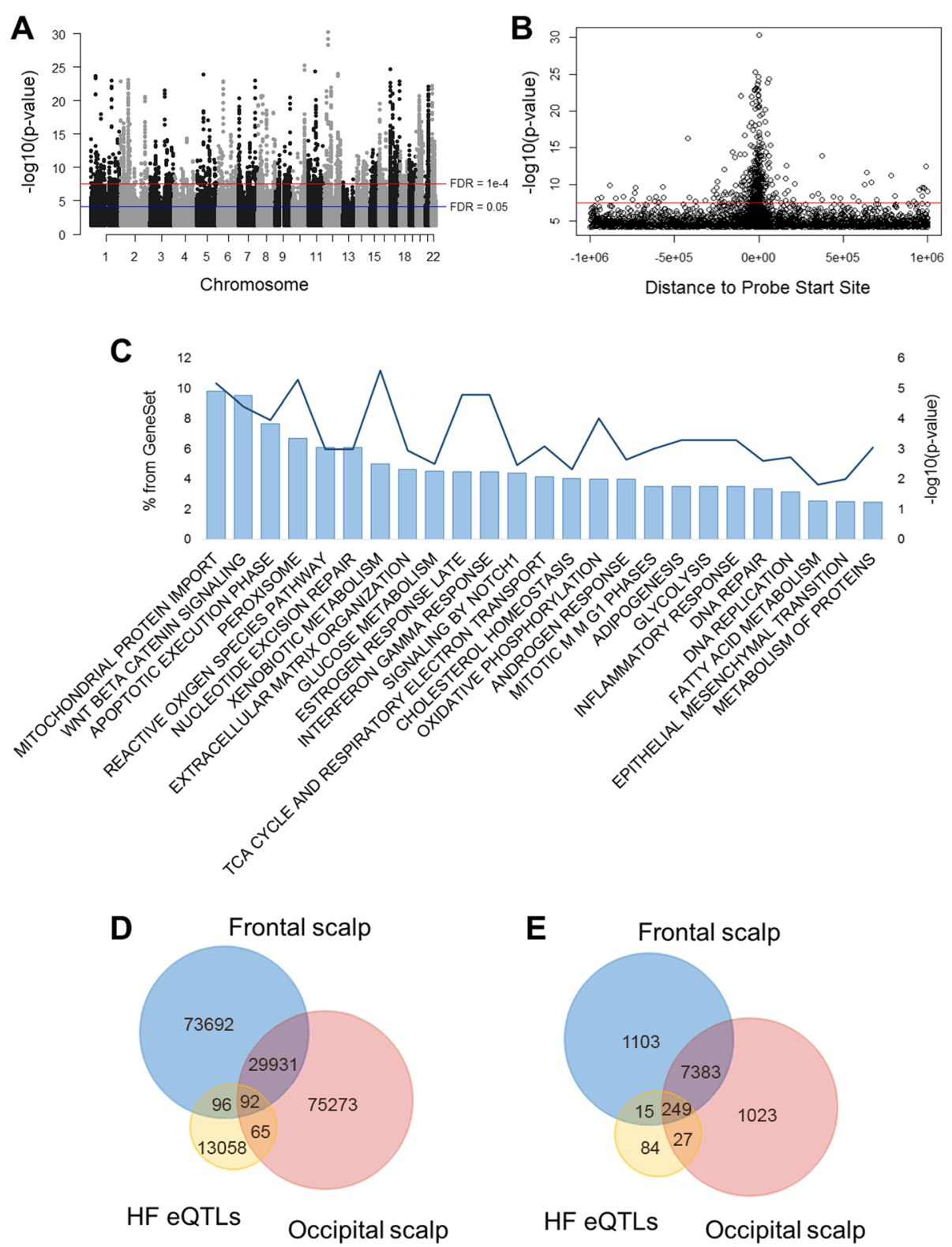

Fig. 1 Hair follicle eQTLs. a Manhattan plot showing all nominally significant $(p<0.05)$ eQTLs. The blue and red lines represent cutoff thresholds at FDR $=0.05$ and $F D R=1 \times 10^{-4}$, respectively. $\mathbf{b}$ Plot showing the $P$-value and distance to probe start site of all top eQTLs (best eSNP per probe) with FDR $<0.05$. The blue line represents the cutoff threshold at FDR $=1 \times 10^{-4}$. $\mathbf{c}$ Representative pathways enriched for the list of eGenes of true eQTLs. $\mathbf{d}$ SNP overlaps between the true hair follicle eQTLs $\left(F D R<1 \times 10^{-4}\right)$ and the relaxed frontal and occipital eQTLs $(p<0.01)$ datasets. e Gene overlaps between the true hair follicle eQTLs and the relaxed frontal and occipital eQTLs datasets

eQTLs (14,497 probe-SNP pairs) (Fig. 1b). From this set of true eQTLs, we identified a total of 374 independent HF eQTLs (Fig. 2a, Suppl.Table.1 [Supplementary Tables]). The top 20 independent eQTL findings are presented in Table 1. Functional enrichment analyses using FUMA revealed that associated eGenes were highly enriched $(F D R<0.05)$ for a variety of Hallmark and Reactome pathways related to metabolism, immune functions, cellular proliferation, apoptosis, adipogenesis and responses to sex hormones (Fig. 1c, Suppl.Table.2 [Supplementary Tables]). Additionally, the annotation of reported eQTL effects with SNiPA showed that 266 of 374 independent eSNPs have been previously reported to have cis-eQTL effects in at least one other tissue, from which 228 were reported to affect the same eGene. Annotation of GWAS Catalog trait associations identified 13 independent HF eSNPs that are associated with 15 traits, including type 2 diabetes mellitus and chronic inflammatory diseases. From the set of true HF eQTLs, 185 eSNPs showed associations with 134 traits, with the highest number of associations found for body mass index (associated 


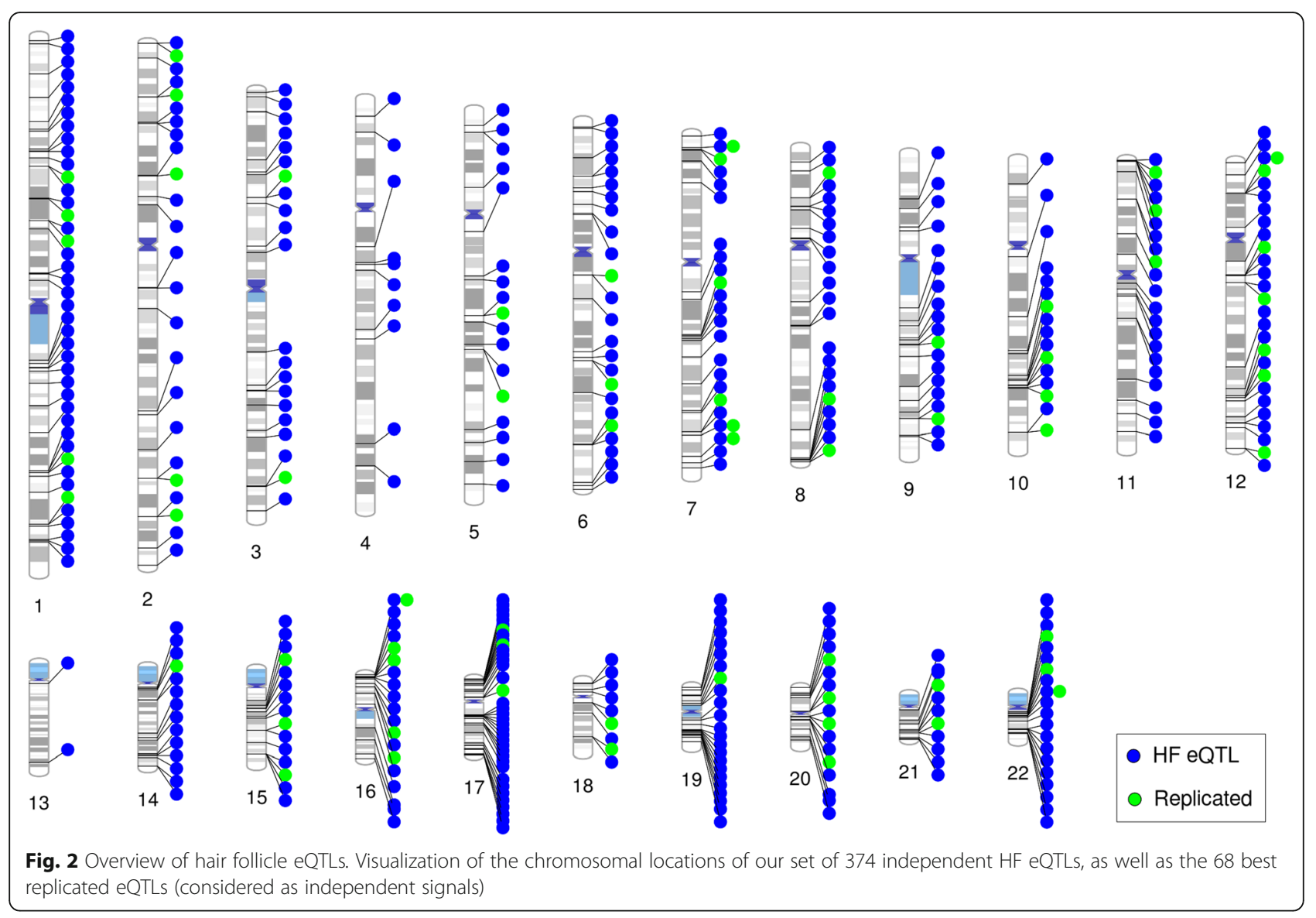

with 29 eSNPs) and blood protein levels (associated with 13 eSNPs) (data not shown).

We achieved the replication of 68 independent signals (Fig. 2) in either occipital and/or frontal scalp HFs from a total of 255 overlapping eQTLs (Suppl.Table.3 [Supplementary Tables]). In total, 188 and 157 eSNPs, and 264 and 276 eGenes, overlapped between our true eQTLs and the frontal and occipital HF eQTLs from the replication sample, respectively. Although only 92 eSNPs overlapped between all three eQTL datasets, these accounted for 249 eGenes (Fig. 1d and e); however, not all eQTL effects were consistent between the three datasets.

\section{Differential HF eQTLs between frontal and occipital scalp areas}

We included all eQTLs with $p<5 \times 10^{-5}$ in the analysis of the replication sample. However, as the power to detect associations is markedly reduced due to the limited sample size $(N=24)$, this is reflected in the number and statistical significance of eQTLs that we detected in frontal and occipital scalp, as well as in their distributions with respect to the probe's TSS (Suppl.Figure.1 [Supplementary Figures]). In general, we found little overlap between these two datasets, and will limit ourselves here to briefly present an overview of regional differences between frontal and occipital scalp.

We identified 71 HF eQTLs with inconsistent effects between frontal and occipital HFs. These were considered "different-effect eQTLs" and affected 11 genes (Suppl.Table.4 [Supplementary Tables]). Furthermore, we identified 289 frontal (Suppl.Table.5 [Supplementary Tables]) and 339 (Suppl.Table.6 [Supplementary Tables]) occipital (region-specific) eQTLs, from which the top 10 for each scalp region are shown in Table 1 . To identify differentiallyenriched pathways, we performed a separate FUMA pathway analysis for frontal and occipital differential eGenes (i.e. region-specific + different-effect) and compared the results for both scalp regions. This analysis suggested differences in the genetically-determined regulation of the responses to steroid hormones, cell cycle control, cellular metabolism and immune functions between the scalp regions (Suppl. Figure.2 [Supplementary figures]). However, as several of the region-specific pathway terms seemed redundant, we sought to further elucidate the differences between both scalp regions through a comparative pathway analysis using FunRich. This analysis suggested that the more important differences between frontal and occipital scalp HFs are on pathways related to the metabolism of amino acids and signaling by histone deacetylases (HDACs) 
Table 1 Top findings for hair follicle eQTLS

\begin{tabular}{|c|c|c|c|c|c|c|c|c|c|}
\hline Gene & \# eQTLs & Best eSNP & Chr & Position & $\mathrm{A} 1$ & $\mathrm{~A} 2$ & $\mathbf{P}$ & FDR & Effect \\
\hline \multicolumn{10}{|c|}{ Top 20 independent eQTLs (discovery sample) } \\
\hline IPO8 & 183 & rs7326 & 12 & $30,782,301$ & G & $A$ & $5.17 \mathrm{E}-31$ & $1.56 \mathrm{E}-24$ & -0.6840 \\
\hline ATP5MD & 95 & rs 2271751 & 10 & $105,175,131$ & C & $\mathrm{T}$ & $5.80 \mathrm{E}-26$ & $3.21 \mathrm{E}-20$ & 1.1004 \\
\hline C17ORF97 & 35 & rs11150881 & 17 & 259,304 & G & A & $2.18 \mathrm{E}-25$ & $1.18 \mathrm{E}-19$ & 0.6600 \\
\hline TIMM10 & 9 & rs3851117 & 11 & $57,237,113$ & A & G & $4.60 \mathrm{E}-25$ & $2.11 \mathrm{E}-19$ & -0.8391 \\
\hline KCTD10 & 211 & rs4766601 & 12 & $109,890,080$ & C & G & $9.89 \mathrm{E}-25$ & 4.49E-19 & 0.5773 \\
\hline C5ORF35/SETD9 & 50 & rs2591963 & 5 & $56,237,135$ & A & $\mathrm{T}$ & 1.50E-24 & $6.43 \mathrm{E}-19$ & -0.6930 \\
\hline NDUFS5 & 45 & rs374279960 & 1 & $39,484,742$ & G & $\mathrm{T}$ & $2.36 \mathrm{E}-24$ & $1.01 \mathrm{E}-18$ & -0.2496 \\
\hline LOC339804 & 267 & rs3213944 & 2 & $61,372,298$ & G & $C$ & $8.36 \mathrm{E}-24$ & $2.50 \mathrm{E}-18$ & 0.4471 \\
\hline FMO1 & 45 & rs6674596 & 1 & $171,235,088$ & $\mathrm{~T}$ & A & $1.04 \mathrm{E}-23$ & $3.07 E-18$ & 1.3913 \\
\hline PARP12 & 32 & rs2286197 & 7 & $139,726,467$ & G & A & $1.19 \mathrm{E}-23$ & 3.46E-18 & 0.3402 \\
\hline SH3YL1 & 50 & rs62114506 & 2 & 242,793 & C & G & $1.30 \mathrm{E}-23$ & $3.78 \mathrm{E}-18$ & -0.5886 \\
\hline PEX6 & 57 & rs3805946 & 6 & $42,955,749$ & $C$ & $T$ & $1.47 \mathrm{E}-23$ & 4.11E-18 & 0.5651 \\
\hline FN3KRP & 109 & rs2249888 & 17 & $80,675,738$ & G & A & $1.54 \mathrm{E}-23$ & $4.11 \mathrm{E}-18$ & 0.3341 \\
\hline$B C R$ & 44 & rs131703 & 22 & $23,652,201$ & G & A & $6.23 \mathrm{E}-23$ & $1.36 \mathrm{E}-17$ & 0.4445 \\
\hline PSMG1 & 295 & rs35064900 & 21 & $40,555,492$ & G & A & $7.92 \mathrm{E}-23$ & $1.55 \mathrm{E}-17$ & -0.3917 \\
\hline RPS26L & 47 & rs773114 & 12 & $56,379,060$ & $\mathrm{~T}$ & A & $1.14 \mathrm{E}-22$ & $2.12 \mathrm{E}-17$ & 0.4386 \\
\hline GOLGBI & 24 & rs9968051 & 3 & $121,384,081$ & G & $C$ & $3.20 \mathrm{E}-22$ & $5.56 \mathrm{E}-17$ & -0.6104 \\
\hline ERP27 & 182 & rs12312821 & 12 & $15,077,527$ & T & A & $4.48 \mathrm{E}-22$ & $7.63 \mathrm{E}-17$ & -0.6385 \\
\hline SLC4TA2 & 51 & rs12451902 & 17 & $19,619,063$ & G & A & $1.02 \mathrm{E}-21$ & $1.65 \mathrm{E}-16$ & 0.6855 \\
\hline ABHD12 & 517 & rs 2258728 & 20 & $25,276,343$ & A & G & $1.91 \mathrm{E}-21$ & $2.88 \mathrm{E}-16$ & 0.2737 \\
\hline \multicolumn{10}{|c|}{ Top 10 eQTLs in frontal scalp (replication sample) } \\
\hline TDRD6 & 14 & rs552053483 & 6 & $46,870,456$ & C & A & $5.32 \mathrm{E}-12$ & 4.08E-06 & 0.4313 \\
\hline CXCL1 & 32 & rs191105830 & 4 & $75,248,687$ & G & A & 3.07E-09 & 7.16E-04 & 0.4703 \\
\hline ZXDC & 12 & rs552704609 & 3 & $126,534,497$ & A & C & 4.70E-08 & 0.00871 & 0.3945 \\
\hline COA4 & 2 & rs34214542 & 11 & $74,207,014$ & G & $\mathrm{T}$ & $7.82 \mathrm{E}-08$ & 0.01141 & 0.3317 \\
\hline DLG2 & 25 & rs141374880 & 11 & $83,245,973$ & A & G & 8.60E-08 & 0.01141 & 0.4126 \\
\hline TADA2A & 3 & rs74398459 & 17 & $36,095,246$ & G & $T$ & $1.17 \mathrm{E}-07$ & 0.01446 & 0.3338 \\
\hline C17orf67 & 2 & rs187188078 & 17 & $55,455,184$ & $\mathrm{~T}$ & $C$ & 1.46E-07 & 0.01761 & 0.6551 \\
\hline FAM $220 A$ & 16 & rs188128204 & 7 & $6,373,651$ & A & G & $2.11 \mathrm{E}-07$ & 0.02083 & 0.4342 \\
\hline ARMC6 & 5 & rs150042710 & 19 & $18,518,809$ & T & $C$ & $2.40 \mathrm{E}-07$ & 0.02262 & 0.6410 \\
\hline NUP153 & 10 & rs115808997 & 6 & $17,571,313$ & $T$ & $C$ & 4.54E-07 & 0.03605 & -0.4850 \\
\hline \multicolumn{10}{|c|}{ Top 10 eQTLs in occipital scalp (replication sample) } \\
\hline TELO2 & 17 & rs561125041 & 16 & $1,464,247$ & T & C & $8.51 \mathrm{E}-10$ & 3.00E-04 & 1.2115 \\
\hline LPHN1 & 17 & rs147147550 & 19 & $14,373,177$ & T & C & $9.90 \mathrm{E}-10$ & $3.00 \mathrm{E}-04$ & 0.6231 \\
\hline ENTHD2 & 5 & rs117860115 & 17 & $78,992,225$ & T & $C$ & 4.10E-09 & 0.00121 & 0.1629 \\
\hline GCSAM & 2 & rs139726887 & 3 & $112,327,159$ & T & C & $3.41 \mathrm{E}-08$ & 0.00496 & 0.3622 \\
\hline CCL2 & 11 & rs72825069 & 17 & $32,600,928$ & T & C & $6.38 \mathrm{E}-08$ & 0.00678 & 1.7221 \\
\hline RUFY3 & 3 & rs115702490 & 4 & $72,452,170$ & T & $C$ & $1.24 \mathrm{E}-07$ & 0.01296 & 0.4149 \\
\hline GGPS1 & 6 & rs115335216 & 1 & $236,067,212$ & $T$ & G & $2.13 \mathrm{E}-07$ & 0.01422 & 0.2215 \\
\hline MTF1 & 10 & rs3935450 & 1 & $37,656,324$ & G & $T$ & $2.65 \mathrm{E}-07$ & 0.01763 & -0.2218 \\
\hline$C X A D R$ & 4 & rs55707799 & 21 & $18,849,240$ & $C$ & $\mathrm{~T}$ & $3.47 \mathrm{E}-07$ & 0.02132 & -0.4272 \\
\hline KRT37 & 1 & rs35490951 & 17 & $40,250,939$ & C & A & 3.65E-07 & 0.02132 & 1.8106 \\
\hline
\end{tabular}

The top 20 hair follicle eQTLs obtained from human occipital scalp in the discovery sample and the top 10 hair follicle eQTLs obtained from frontal and occipital scalp in the replication sample are shown 
in the frontal scalp, and to proliferative processes in the occipital area (Table 2).

\section{Overlaps with genomic regions associated with hair phenotypes}

To illustrate the applicability of our set of true eQTLs to interpret GWAS findings for hair phenotypes, we investigated for a potential overlap of HF eSNPs with genomic risk loci for AGA (98 loci), hair shape (12 loci) and hair color (122 loci) (Table 3). We identified one genomic locus at $1 \mathrm{p} 36.13$ that had previously been associated with hair phenotypes. This comprised 31 eSNPs overlapping association signals for hair shape (overlapping lead SNP: rs11203346) and hair color (overlapping lead SNP: rs72646785) (Fig. 3a). For this locus, the nearest genes were mapped to PADI3 and PADI4 in both GWAS, while the eGene for this region corresponded to PADI3. Moreover, another locus at 4q21.21, comprised by 26 eSNPs linked to the signal of rs6533756 (a noneSNP in LD $r^{2} \geq 0.8$ with all eSNPs), was associated with hair shape. The overlapping eSNP most strongly associated with hair shape at this locus was $\operatorname{rs7695038}(p=$ $\left.3.9 \times 10^{-10}\right)$. While eSNPs at this locus locate within a region coding for the FRAS1 gene, the associated eGene, $A N X A 3$, was located $66,182 \mathrm{bp}$ downstream of FRAS1 (Fig. 3b). A third locus at 17q25.3 comprised the eSNP, rs8070929, which was in LD $\left(r^{2} \geq 0.97\right)$ with rs34872037 (a non-eSNP) that is associated with hair color. While the nearest gene for the SNP at this locus was NPLOC4, the HF eGene corresponded to TSPAN10 (Fig. 3c). All eSNPs overlapping association signals for hair shape and/or hair color can be found in Suppl.Table.7. We found no overlaps of our true HF eQTL loci with the reported genome-wide significant findings for AGA. However, we observed that the eGene ATP2B4 was annotated as the nearest gene to an AGA genomic locus at $1 \mathrm{q} 32.1$. Moreover, it is perhaps worth to notice that, at the nominal significance level $(p<0.05)$, we found 93 eGenes to overlap with reported AGA candidate genes, of which 18 were associated with eSNPs at an FDR < 0.05 (ALPL, B3GNT8, BCL2, DIP2B, FAM136A, HDAC9, LCLAT1, LHPP, NSF, PRRX1, RPTN, RSPO2, SUCNR1, TCHH, TMEM5OA, TWIST2, MSIG2 and SPAG17).

\section{Discussion}

The strongest eQTL associations in occipital HFs were observed for IPO8 (rs7326), ATP5MD (rs2271751), and C17orf97/LIAT1 (rs11150881). The findings for ATP5MD were confirmed in our small replication study. Although we were not able to replicate the "true" SNP-gene associations for IPO8 in our replication sample, a set of different SNPs were indeed associated with IPO8 expression in HFs from frontal and/or occipital scalp areas, some of which were also associated with IPO8 expression in the discovery

Table 2 Best frontal- and occipital-specific biological pathways enriched for differential eGenes

\begin{tabular}{|c|c|c|c|c|c|c|c|}
\hline \multirow[t]{2}{*}{ Biological pathway } & \multirow[t]{2}{*}{ GeneSet } & \multicolumn{3}{|c|}{ Frontal scalp } & \multicolumn{3}{|c|}{ Occipital scalp } \\
\hline & & n & Fold value & $\mathbf{P}$ & $\mathbf{n}$ & Fold value & $\mathbf{P}$ \\
\hline \multicolumn{8}{|l|}{ Frontal scalp eQTLs } \\
\hline Activation of Chaperones by ATF6-alpha & 9 & 3 & 21.85 & $2.78 \mathrm{E}-04$ & 0 & 0.01 & 1 \\
\hline Interconversion of 2-oxoglutarate and 2-hydroxyglutarate & 3 & 2 & 43.68 & $6.92 \mathrm{E}-04$ & 0 & 0.01 & 1 \\
\hline Pyruvate metabolism and Citric Acid (TCA) cycle & 31 & 4 & 8.46 & $1.22 \mathrm{E}-03$ & 0 & 0.01 & 1 \\
\hline Arginine degradation I (arginase pathway) & 4 & 2 & 32.78 & 1.37E-03 & 0 & 0.01 & 1 \\
\hline Proline biosynthesis II (from arginine) & 5 & 2 & 26.24 & $2.26 \mathrm{E}-03$ & 0 & 0.01 & 1 \\
\hline Arginine degradation VI (arginase 2 pathway) & 6 & 2 & 21.87 & $3.36 \mathrm{E}-03$ & 0 & 0.01 & 1 \\
\hline Vitamin C (ascorbate) metabolism & 6 & 2 & 21.87 & $3.36 \mathrm{E}-03$ & 0 & 0.01 & 1 \\
\hline Dolichyl-diphosphooligosaccharide biosynthesis & 7 & 2 & 18.75 & $4.65 \mathrm{E}-03$ & 0 & 0.01 & 1 \\
\hline Signaling events mediated by HDAC Class I & 113 & 6 & 3.48 & 7.84E-03 & 1 & 0.51 & $8.66 \mathrm{E}-01$ \\
\hline Signaling events mediated by HDAC Class II & 38 & 4 & 6.90 & $2.62 \mathrm{E}-03$ & 1 & 1.51 & 4.91E-01 \\
\hline BMP receptor signaling & 226 & 9 & 2.61 & $8.19 \mathrm{E}-03$ & 5 & 1.26 & $3.66 \mathrm{E}-01$ \\
\hline Amino acid synthesis and interconversion (transamination) & 10 & 3 & 19.67 & $3.92 \mathrm{E}-04$ & 1 & 5.74 & $1.63 \mathrm{E}-01$ \\
\hline \multicolumn{8}{|l|}{ Occipital scalp eQTLs } \\
\hline BARD1 signaling events & 29 & 1 & 2.28 & 3.61E-01 & 4 & 7.86 & 1.57E-03 \\
\hline G0 and Early G1 & 21 & 0 & 0.01 & 1 & 3 & 8.15 & $5.66 \mathrm{E}-03$ \\
\hline TGFBR & 125 & 4 & 2.10 & $1.25 \mathrm{E}-01$ & 7 & 3.19 & 6.64E-03 \\
\hline VEGF and VEGFR signaling network & 1301 & 27 & 1.36 & $6.57 \mathrm{E}-02$ & 35 & 1.53 & 8.04E-03 \\
\hline
\end{tabular}


Table 3 Genomic loci for hair phenotypes and their observed overlaps with our hair follicle eQTLS

\begin{tabular}{|c|c|c|c|}
\hline Summary & Hair shape & Hair color & AGA \\
\hline Sample size & 16,763 & 290,891 & $145,000^{\circ}$ \\
\hline \# Genomic loci & 12 & 122 & 98 \\
\hline \# Lead SNPs & 23 & 124 & 185 \\
\hline \# Ind.Sig.SNPs & 63 & 130 & 245 \\
\hline \# Candidate SNPs & 1424 & 6658 & 11,821 \\
\hline \# GWAS SNPS & 704 & 134 & 335 \\
\hline \# Mapped genes & 69 & 204 & 161 \\
\hline \# Total input SNPs & 706 & 137 & 387 \\
\hline \# Skipped input SNPS & 2 & 3 & 36 \\
\hline Overlapping eGenes with mapped genes & PADI3 & BCAS1, C17orf70, CHMP4C, MRPL39, PADI3, RYBP & ATP2B4 \\
\hline Overlapping loci & 2 & 2 & - \\
\hline \multirow[t]{2}{*}{ Overlapping Chr.Loc. } & chr1p36.13 & chr1p36.13 & - \\
\hline & chr4q21.21 & chr17q25.3 & \\
\hline \multirow[t]{2}{*}{ Mapped genes } & PADI3, PAD/4 & PADI3, PADI4 & - \\
\hline & FRAS1 & NPLOC4 & \\
\hline \multirow[t]{2}{*}{ eGenes } & PADI3 & PADI3 & - \\
\hline & ANXA3 & TSPAN10 & \\
\hline
\end{tabular}

AGA androgenetic alopecia, Ind.Sig.SNPs independent significant SNPs, Chr.Loc. chromosome location

${ }^{a}$ Approximated sample size, as lead SNPs were collected from different studies

sample at the nominal level (data not shown), therefore confirming the genetic regulation of IPO8 in HFs. While little is known about the function of C17orf97, IPO8 mediates the nuclear import of proteins and mature microRNAs [28]. ATP5MD is crucial for the maintenance of ATP synthase in mitochondria, and might actively participate in the cellular energy metabolism, a process with well-known relevance to hair biology and hair growth $[29,30]$. Overexpression of ATP5MD causes a number of mitochondrial abnormalities and an increase in anaerobic metabolism associated with the induction of an epithelial to mesenchymal-like transition, as well as delayed cell growth $[31,32]$. The genetically-controlled regulation of mitochondrial function in human HF is further supported by our pathway analysis that found an enrichment of eGenes for true eQTLs in pathways related to mitochondrial function along with other pathways, such as the regulation of responses to steroid hormones, the Wnt $/ \beta$-catenin and interferon (IFN) signaling, adipogenesis, immune responses and the metabolism of glucose and lipids, all of which have well-known roles in HF biology [33].

We also investigated whether there might be differences in the genetic control of gene expression between HF subpopulations from different scalp areas. Despite the reduced size of this (replication) sample, our results point to interesting avenues for future research. For instance, an important difference between frontal and occipital scalp appears to be the metabolism of amino acids, including arginine degradation. It is known that the HF is dependent on arginine, as hair growth depends on the vasculature and L-arginine not only participates in cell proliferation but is a precursor for the vascular mediator nitric oxide. L-arginine deficiency has been shown to impair hair elongation, while its supplementation increases the number of HFs in anagen (growth) and decreases that of HFs in telogen (resting) phase [33, 34]. This supports the notion that regional increases in arginine degradation might result in enhanced vulnerability to hair loss in the frontal scalp area. A similar scenario can be thought for HDACs. HDACs are important transcriptional repressors that act in multiprotein complexes and are involved in the control of cell cycle progression $[35,36]$. While class I HDACs are ubiquitously expressed, class II HDACs show tissue specificity [36]. In our study, we found eQTLs for HDACs 2, 5 and 7 only in frontal scalp. Interestingly, these particular HDACs modulate HF development and homeostasis [37, 38], as well as angiogenesis and vascular integrity [39-41]. Moreover, androgen actions have been shown to regulate HDAC7 subcellular compartmentalization, and HDAC7 has been proposed to be a co-repressor of the androgen receptor [42].

We also found evidence for differential regulation of vitamin $\mathrm{C}$ (L-ascorbic acid) metabolism in frontal scalp. It has been shown that a derivative of $\mathrm{L}$-ascorbic acid (L-ascorbic acid 2-phosphate) promotes HF growth that is mediated by the induced expression of insulin-like growth factor-1 (IGF-1) in dermal papilla cells [43]. This opens the possibility that decreased expression of genes involved in the metabolism of L-ascorbic acid in frontal 

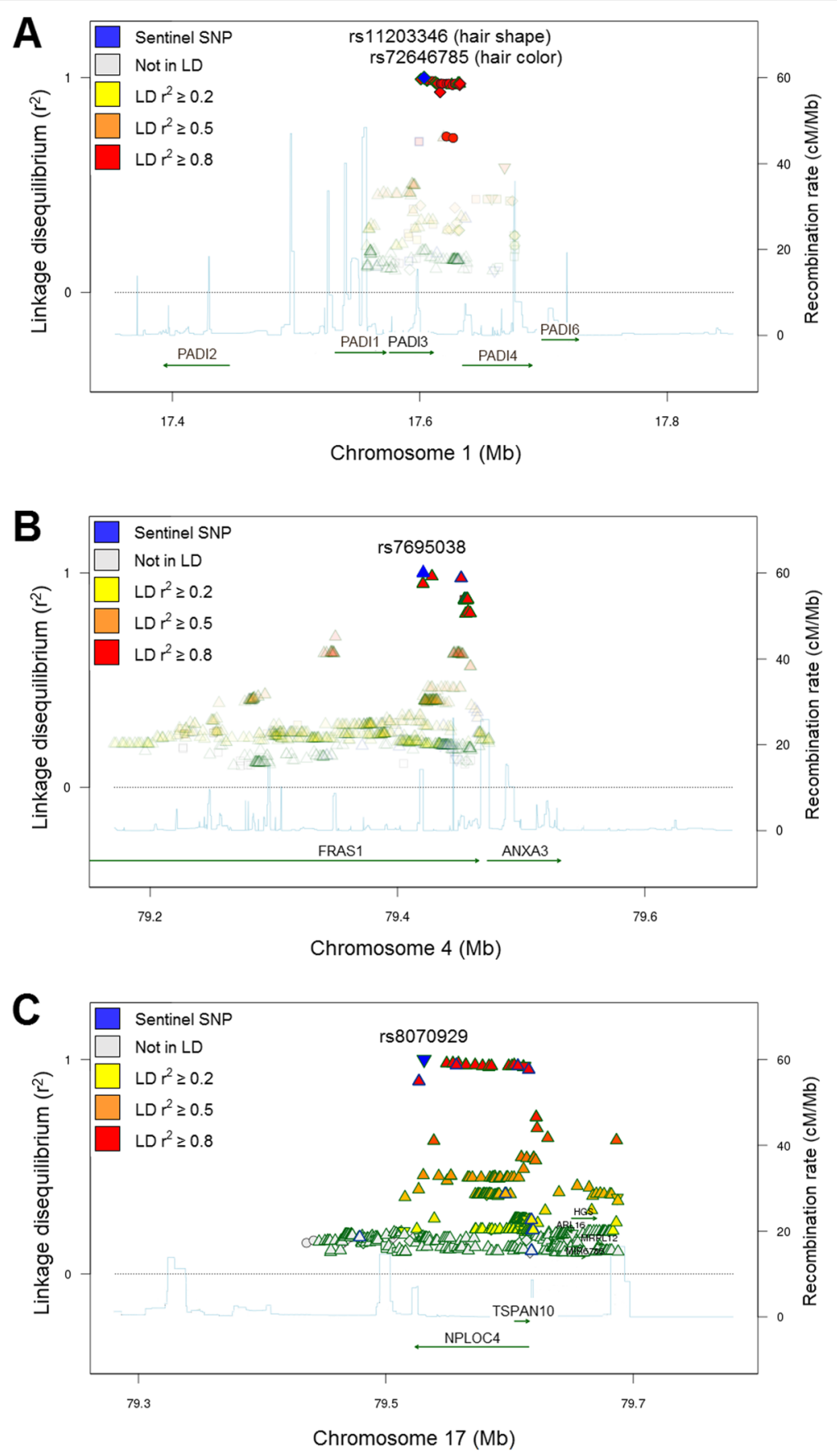

Fig. 3 Hair follicle eQTLs overlapping genetic findings for hair-related traits. Linkage disequilibrium plots highlighting: a 31 HF eQTLs that overlapped a genomic locus shared by hair shape and hair color, b $26 \mathrm{HF}$ eQTLs that overlapped a genomic locus for hair shape, with the most strongly associated SNP used as sentinel (in blue), and c one HF eQTL (blue) that overlapped a genomic locus for hair color. GWAS loci were defined through an analysis with the FUMA SNP2GENE function, applied separately to the genome-wide findings of GWAS for hair shape and hair color. The plots were generated using SNiPA. For the plot in a, rs72646785 (independent HF eQTL for the region) was used as the sentinel SNP (blue). The lead SNPs within this locus for hair shape (rs1 1203346) and hair color (rs72646785) are separated from each other by $2650 \mathrm{bp}$ and have an LD $r^{2}=0.99$ in the $1000 \mathrm{Genomes}$ Project, Phase 3 v5, European population reference panel

scalp might render this region more susceptible to hair loss. Taken together, our pathway analysis results show that frontal-specific pathways present several factors with negative effects on hair growth (e.g. androgen and estrogen responses, BMP2/4 signaling, IFN- $\gamma$ response, endocannabinoid signaling), while occipital-specific pathways are more consistent with factors exerting positive effects on hair growth (e.g. vascular endothelial 
growth factor signaling, transforming growth factor beta receptor), considering what Bernard has referred to as "the Yin Yang of the human hair follicle" [33]. Nevertheless, the implications of genetic regulation in frontal scalp for hair loss disorders should be investigated through the generation of a confident eQTL dataset with increased power in future studies.

With our study, we also show that tissue-specific eQTL data are a valuable resource to identify regulatory effects at disease- and trait-associated loci. In particular, our results suggest an important role of PADI3 in hair traits. Indeed, PADI3 is located in the inner root sheath and medulla in anagen HFs and has been reported to play roles in HF differentiation [44] and hair shaft formation [45]. Our results also implicate $A N X A 3$ and TSPAN10 as novel candidate genes for hair shape and color, respectively. Although we found no overlap between the true HF eQTLs and AGA genetic risk loci, which might be an expected finding, considering that the occipital scalp is not susceptible to balding, the overlapping eGene ATP2B4 provides a potential novel candidate gene for AGA, as the reported gene for the region is so far $\operatorname{SOX} 13[25,26]$.

The most obvious limitation of our study is the sample size. However, we tried to overcome this limitation by applying high quality standards to the data and stringent selection criteria to our HF eQTL results. Another potential limitation of our study resides in the use of plucked HFs instead of intact HFs. However, a skin biopsy is required in order to obtain intact HFs, whereas hair plucking is a less invasive technique. Moreover, it has been demonstrated that plucked hairs retain most epithelial structures, maintain the integrity of the outer root sheet and also contain stem cells [46]. Finally, due to the small size of our samples, particularly that of the replication sample, we considered advisable to exclude from the present study indels and sex chromosomes, and limit ourselves to reporting, in very general terms, differential findings between HFs from frontal and occipital scalp areas.

\section{Conclusions}

Our analyses demonstrate that well-established HF molecular pathways are genetically regulated and that, to some extent, this regulation can show regional specificity within scalp HFs. The enriched pathways mainly underscored processes that relate to hair growth and the HF cycle. More detailed tissuespecific analyses will be enabled by future increases in sample size, ensuring an improved understanding of the genetically determined variability in HF gene expression and its implications for hair-related traits and hair loss disorders.

\section{Supplementary Information}

The online version contains supplementary material available at https://doi. org/10.1186/s12895-020-00113-y.

Additional file 1. Supplementary Tables.

Additional file 2. Supplementary Figures.

\section{Abbreviations}

AGA: Androgenetic alopecia; eQTLs: Expression quantitative trait loci; FDR: False discovery rate; FUMA: Functional mapping and annotation of GWAS; GWAS: Genome-wide association analysis; HF(s): Hair follicle(s); HDACs: Histone deacetylases; IFN: Interferon; LD: Linkage disequilibrium; MAF: Minor allele frequency; PCA: Principal component analysis; PCs: Principal components; RIN: RNA integrity number; SNP: Single nucleotide polymorphism; TSS: Transcription start site

\section{Acknowledgments}

The authors thank the study participants for their cooperation. MMN and MHR are members of the Excellence Cluster ImmunoSensation.

\section{Authors' contributions}

MHR contributed to the design of the study, performed the analyses and prepared the manuscript. LMH participated in data collection. SS provided support with the initial eQTL analysis. MMN participated in the conception of the study and provided critical revisions. SHH conceived and designed the study, participated in the data collection, provided critical revisions to the analyses, and prepared and revised the manuscript. The author(s) read and approved the final manuscript.

\section{Funding}

The study was supported by the BONFOR program of the Medical Faculty of the University of Bonn. Open Access funding enabled and organized by Projekt DEAL.

\section{Availability of data and materials}

The datasets used for this study are available from the corresponding author on reasonable request. The derived data supporting the conclusions of this article are included within the article and its additional files.

\section{Ethics approval and consent to participate}

All participants provided written informed consent. The study was approved by the Institutional Ethics Review Committee of the University of Bonn and experiments were conducted in accordance with the Declaration of Helsinki.

Consent for publication

'Not applicable'

\section{Competing interests}

The authors declare that they have no competing interests.

\section{Author details}

${ }^{1}$ Institute of Human Genetics, University of Bonn, School of Medicine \& University Hospital Bonn, 53127 Bonn, Germany. ${ }^{2}$ Present address: Department of Genetic Epidemiology, Institute of Human Genetics, University of Münster, 48149 Münster, Germany.

Received: 25 April 2019 Accepted: 30 October 2020

Published online: 10 November 2020

References

1. Liu F, Chen Y, Zhu G, Hysi PG, Wu S, Adhikari K, et al. Meta-analysis of genome-wide association studies identifies 8 novel loci involved in shape variation of human head hair. Hum Mol Genet. 2018;27(3):559-75.

2. Hysi PG, Valdes AM, Liu F, Furlotte NA, Evans DM, Bataille V, et al. Genomewide association meta-analysis of individuals of European ancestry identifies new loci explaining a substantial fraction of hair color variation and heritability. Nat Genet. 2018;50(5):652-6.

3. Chen L, Qin ZS. Using DIVAN to assess disease/trait-associated single nucleotide variants in genome-wide scale. BMC Res Notes. 2017;10(1):530. 
4. Gilad Y, Rifkin SA, Pritchard JK. Revealing the architecture of gene regulation: the promise of eQTL studies. Trends Genet. 2008;24(8):408-15.

5. Hochfeld LM, Anhalt T, Reinbold CS, Herrera-Rivero M, Fricker N, Nöthen MM, Heilmann-Heimbach S. Expression profiling and bioinformatic analyses suggest new target genes and pathways for human hair follicle related microRNAs. BMC Dermatol. 2017:17(1):3.

6. Chang CC, Chow CC, Tellier LC, Vattikuti S, Purcell SM, Lee JJ. Secondgeneration PLINK: rising to the challenge of larger and richer datasets. Gigascience. 2015;4:7.

7. Das S, Forer L, Schönherr S, Sidore C, Locke AE, Kwong A, et al. Nextgeneration genotype imputation service and methods. Nat Genet. 2016; 48(10):1284-7.

8. Loh PR, Danecek P, Palamara PF, Fuchsberger C, Reshef AY, Finucane KH, et al. Reference-based phasing using the haplotype reference consortium panel. Nat Genet. 2016:48(11):1443-8.

9. Danecek P, Auton A, Abecasis G, Albers CA, Banks E, DePristo MA, et al. The variant call format and VCFtools. Bioinformatics. 2011;27(15):2156-8.

10. Dunning $M$, Lynch A, Eldridge M. illuminaHumanv4.db: Illumina HumanHT12v4 annotation data (chip illuminaHumanv4). R package version 1.26.0; 2015.

11. Delaneau $\mathrm{O}$, Ongen $\mathrm{H}$, Brown AA, Fort A, Panousis NI, Dermitzakis ET. A complete tool set for molecular QTL discovery and analysis. Nat Commun. 2017;8:15452.

12. Arnold M, Raffler J, Pfeufer A, Suhre K, Kastenmüller G. SNiPA: an interactive, genetic variant-centered annotation browser. Bioinformatics. 2015;31(8): 1334-6 Available at http://www.snipa.org. Accessed Oct 2018.

13. MacArthur J, Bowler E, Cerezo M, Gil L, Hall P, Hastings E, et al. The new NHGRI-EBI catalog of published genome-wide association studies (GWAS catalog). Nucleic Acids Res. 2017;45(D1):D896-901.

14. Watanabe K, Taskesen E, van Bochoven A, Posthuma D. Functional mapping and annotation of genetic associations with FUMA. Nat Commun. 2017;8(1):1826.

15. Pathan M, Keerthikumar S, Ang CS, Gangoda L, Quek CY, Williamson NA et al. FunRich: an open access standalone functional enrichment and interaction network analysis tool. Proteomics. 2015;15(15):2597-601.

16. Hillmer AM, Flaquer A, Hanneken S, Eigelshoven S, Kortüm AK, Brockschmidt FF, et al. Genome-wide scan and fine-mapping linkage study of androgenetic alopecia reveals a locus on chromosome 3q26. Am J Hum Genet. 2008;82(3):737-43.

17. Richards JB, Yuan X, Geller F, Waterworth D, Bataille V, Glass D, et al. Malepattern baldness susceptibility locus at 20p11. Nat Genet. 2008;40(11):1282-4.

18. Brockschmidt FF, Heilmann S, Ellis JA, Eigelshoven S, Hanneken S, Herold C, et al. Susceptibility variants on chromosome 7p21.1 suggest HDAC9 as a new candidate gene for male-pattern baldness. Br J Dermatol. 2011;165(6):1293-302.

19. Li R, Brockschmidt FF, Kiefer AK, Stefansson H, Nyholt DR, Song K, et al. Six nove susceptibility loci for early-onset androgenetic alopecia and their unexpected association with common diseases. PLoS Genet. 2012;8(5):e1002746.

20. Adhikari K, Fontanil T, Cal S, Mendoza-Revilla J, Fuentes-Guajardo M, Chacón-Duque JC, et al. A genome-wide association scan in admixed Latin Americans identifies loci influencing facial and scalp hair features. Nat Commun. 2016;7:10815.

21. Liu F, Hamer MA, Heilmann S, Herold C, Moebus S, Hofman A, et al. Prediction of male-pattern baldness from genotypes. Eur J Hum Genet. 2016;24(6):895-902.

22. Pickrell JK, Berisa T, Liu JZ, Ségurel L, Tung JY, Hinds DA. Detection and interpretation of shared genetic influences on 42 human traits. Nat Genet. 2016;48(7):709-17.

23. Heilmann S, Kiefer AK, Fricker N, Drichel D, Hillmer AM, Herold C, et al. Androgenetic alopecia: identification of four genetic risk loci and evidence for the contribution of WNT signaling to its etiology. J Invest Dermatol. 2013;133(6):1489-96.

24. Heilmann-Heimbach S, Herold C, Hochfeld LM, Hillmer AM, Nyholt DR, Hecker $J$, et al. Meta-analysis identifies novel risk loci and yields systematic insights into the biology of male-pattern baldness. Nat Commun. 2017:8:14694.

25. Hagenaars SP, Hill WD, Harris SE, Ritchie SJ, Davies G, Liewald DC, et al. Genetic prediction of male pattern baldness. PLoS Genet. 2017;13(2):e1006594.

26. Pirastu N, Joshi PK, de Vries PS, Cornelis MC, McKeigue PM, Keum N, et al. GWAS for male-pattern baldness identifies 71 susceptibility loci explaining 38\% of the risk. Nat Commun. 2017;8(1):1584.

27. 1000 Genomes Project Consortium, Auton A, Brooks LD, Durbin RM, Garrison EP, Kang HM, et al. A global reference for human genetic variation. Nature. 2015;526(7571):68-74.
28. Wei Y, Li L, Wang D, Zhang CY, Zen K. Importin 8 regulates the transport of mature microRNAs into the cell nucleus. J Biol Chem. 2014;289(15):10270-5.

29. Vidali S, Knuever J, Lerchner J, Giesen M, Bíró T, Klinger M, et al. Hypothalamic-pituitary-thyroid axis hormones stimulate mitochondrial function and biogenesis in human hair follicles. J Invest Dermatol. 2014; 134(1):33-42.

30. Singh B, Schoeb TR, Bajpai P, Slominski A, Singh KK. Reversing wrinkled skin and hair loss in mice by restoring mitochondrial function. Cell Death Dis. 2018;9(7):735

31. Ohsakaya S, Fujikawa M, Hisabori T, Yoshida M. Knockdown of DAPIT (diabetes-associated protein in insulin-sensitive tissue) results in loss of ATP synthase in mitochondria. J Biol Chem. 2011;286(23):20292-6.

32. Kontro H, Cannino G, Rustin P, Dufour E, Kainulainen H. DAPIT overexpression modulates glucose metabolism and cell behaviour in HEK293T cells. PLoS One. 2015:10(7):e0131990.

33. Bernard BA. Advances in understanding hair growth. F1000Res. 2016;5:F1000 Faculty Rev-147.

34. Michelet JF, Bernard BA, Juchaux F, Michelin C, El Rawadi C, Loussouarn G, Pereira R. Importance of L-Arginine for human hair growth, 28th IFSCC Meeting Proceedings; 2014. p. 1123-8.

35. Verdin E, Dequiedt F, Kasler HG. Class II histone deacetylases: versatile regulators. Trends Genet. 2003:19(5):286-93.

36. Gallinari $P$, Di Marco S, Jones P, Pallaoro M, Steinkühler C. HDACs, histone deacetylation and gene transcription: from molecular biology to cancer therapeutics. Cell Res. 2007;17(3):195-211.

37. LeBoeuf M, Terrell A, Trivedi S, Sinha S, Epstein JA, Olson EN, et al. Hdac and Hdac2 act redundantly to control p63 and p53 functions in epidermal progenitor cells. Dev Cell. 2010;19(6):807-18.

38. Hughes MW, Jiang TX, Lin SJ, Leung Y, Kobielak K, Widelitz RB, Chuong CM. Disrupted ectodermal organ morphogenesis in mice with a conditional histone deacetylase 1, 2 deletion in the epidermis. J Invest Dermatol. 2014; 134(1):24-32.

39. Mottet D, Bellahcène A, Pirotte $S$, Waltregny $D$, Deroanne $C$, Lamour V, et al. Histone deacetylase 7 silencing alters endothelial cell migration, a key step in angiogenesis. Circ Res. 2007;101(12):1237-46.

40. Urbich C, Rössig L, Kaluza D, Potente M, Boeckel JN, Knau A, et al. HDAC5 is a repressor of angiogenesis and determines the angiogenic gene expression pattern of endothelial cells. Blood. 2009;113(22):5669-79.

41. Trivedi CM, Zhu W, Wang Q, Jia C, Kee HJ, Li L, et al. Hopx and Hdac2 interact to modulate Gata4 acetylation and embryonic cardiac myocyte proliferation. Dev Cell. 2010;19(3):450-9.

42. Karvonen $\mathrm{U}$, Jänne $\mathrm{OA}$, Palvimo JJ. Androgen receptor regulates nuclear trafficking and nuclear domain residency of corepressor HDAC7 in a liganddependent fashion. Exp Cell Res. 2006;312(16):3165-83.

43. Kwack MH, Shin SH, Kim SR, Im SU, Han IS, Kim MK, et al. I-Ascorbic acid 2phosphate promotes elongation of hair shafts via the secretion of insulinlike growth factor-1 from dermal papilla cells through phosphatidylinositol 3-kinase. Br J Dermatol. 2009:160(6):1157-62

44. Chavanas S, Méchin MC, Nachat R, Adoue V, Coudane F, Serre G, Simon M. Peptidylarginine deiminases and deimination in biology and pathology: relevance to skin homeostasis. J Dermatol Sci. 2006;44(2):63-72.

45. Basmanav ÜFB, Cau L, Tafazzoli A, Méchin MC, Wolf S, Romano MT, et al. Mutations in three genes encoding proteins involved in hair shaft formation cause uncombable hair syndrome. Am J Hum Genet. 2016;99(6):1292-304.

46. Schembri K, Scerri C, Ayers D. Plucked human hair shafts and biomolecular medical research. ScientificWorldJournal. 2013;2013:620531.

\section{Publisher's Note}

Springer Nature remains neutral with regard to jurisdictional claims in published maps and institutional affiliations. 